\title{
Ondes asymptotiques non linéaires $\left(^{*}\right)$.
}

\author{
GUY BoILLAT (Clermont-Ferrand)
}

A Dario Graffi à l'occasion de son 70-ème anniversaire

Résumé. - Le terme du premier ordre de l'onde asymptotique a une forme particulièrement simple non seulement quand la solution de base est constante mais aussi, en ce qui concerne sa norme, lorsque les équations dérivent d'un principe variationnel. Par un changement des variables de champ les équations d'écalution d'Euler s'écrivent comme un système symétrique du premier ordre avec second membre antisymétrique dont toutes les matrices sont constantes sauf celle qui multiplie la dérivée temporelle et qui est un double gradient.

Un champ $u\left(x^{\alpha}\right)(\alpha=0,1,2, \ldots, n)$ fonction $d u$ temps $\left(x^{0}=t\right)$ et des variables d'espace $x^{i}(i=1,2, \ldots, n)$ satisfait à un ensemble d'équations aux dérivées partielles ramené à un système du premier ordre quasi linéaire [1],

$$
A^{\alpha}\left(u, x^{\beta}\right) u_{\alpha}=f\left(u, x^{\beta}\right), \quad A^{0}=I,
$$

où les $A^{\alpha}$ sont des matrices carrées $N \times N$.

Nous poserons

$$
u=u_{0}\left(x^{\alpha}\right)+\frac{1}{\omega} u_{1}\left(x^{\alpha} ; \xi\right)+\frac{1}{\omega^{2}} u_{2}\left(x^{\alpha} ; \xi\right)+\ldots
$$

suivant J. LeRAY, L. GÅRDIng et T. Kotake [2] qui ont traité le cas linéaire en généralisant les développements de P. D. Lax et D. Ludwig [3], [4] et Mme Y. ChoQUET [5] qui a étudié le cas non linéaire. $u_{0}\left(x^{\alpha}\right)$ est une solution de (1), $\omega$ une constante et $\xi=\omega \varphi$ où $\varphi\left(x^{\alpha}\right)$, la phase, est une fonction à déterminer.

On voit immédiatement que les ondes de discontinuités seront obtenues formellement en choisissant

$$
u_{p}\left(x^{\alpha} ; \xi\right)=\frac{\xi^{p}}{p !} \pi_{p}\left(x^{\alpha}\right)
$$

et en supposant que (2) est valable d'un côté de la surface d'onde $(\varphi>0)$ tandis que de l'autre côté $(\varphi<0)$ on aura simplement $u=u_{0}$.

(*) Entrata in Redazione il 15 giugno 1975. 
On a,

$$
\begin{aligned}
& A^{\alpha}\left(u, x^{\beta}\right)=A^{\alpha}\left(u_{0}, x^{\beta}\right)+\frac{1}{\omega} \nabla A^{\alpha}\left(u_{0}, x^{\beta}\right) u_{1}+O\left(\frac{1}{\omega^{2}}\right), \\
& \partial_{\alpha} u=u_{0_{\alpha}}+\frac{1}{\omega}\left(\frac{\partial u_{1}}{\partial x^{\alpha}}+\omega \frac{\partial u_{1}}{\partial \xi} \varphi_{\alpha}\right)+\frac{1}{\omega} \frac{\partial u_{2}}{\partial \xi} \varphi_{\alpha}+O\left(\frac{1}{\omega^{2}}\right), \\
& f\left(u, x^{\alpha}\right)=f\left(u_{0}, x^{\alpha}\right)+\frac{1}{\omega} \nabla f\left(u_{0}, x^{\alpha}\right) u_{1}+O\left(\frac{1}{\omega^{2}}\right)
\end{aligned}
$$

où $\varphi_{\alpha}=\partial_{\alpha} \varphi$ et le nabla est le gradient par rapport aux composantes de $u$. On en déduit en portant dans (1),

$$
\begin{aligned}
& A_{0}^{\alpha} \varphi_{\alpha} \frac{\partial u_{1}}{\partial \xi}=0, \quad A_{0}^{\alpha}=A^{\alpha}\left(u_{0}, x^{\beta}\right), \\
& A_{0}^{\alpha}\left(\frac{\partial u_{1}}{\partial x^{\alpha}}+\varphi_{\alpha} \frac{\partial u_{2}}{\partial \xi}\right)+\nabla A_{0}^{\alpha} u_{1}\left(u_{0_{\alpha}}+\frac{\partial u_{1}}{\partial \xi} \varphi_{\alpha}\right)=\nabla f_{0} u_{1} .
\end{aligned}
$$

Définissons le vecteur normal unitaire $n\left(x^{\alpha}\right)$ de composantes $n_{i}=\varphi_{i} /$ grad $\varphi \mid$, $\sum n_{i}^{2}=1$. Le système (1) est hyperbolique si la matrice $A_{n}=A^{i} n_{i}$ admet $N$ vecteurs propres linéairement indépendants correspondant à des valeurs propres $\lambda\left(u, x^{\alpha} ; n\right)$ réelles. On distingue les vecteurs propres droits et gauches, $d\left(u, x^{\alpha} ; n\right), l\left(u, x^{\alpha} ; n\right)$,

$$
\left(A_{n}-\lambda I\right) d_{I}=0, \quad l_{I^{\prime}}\left(A_{n}-\lambda I\right)=0, \quad I, I^{\prime}=1,2, \ldots, m
$$

où $\lambda$ est de multiplicité $m$.

La solution de l'équation (3) s'écrit,

$$
u_{1}=u^{I}\left(x^{\alpha} ; \xi\right) d_{I}\left(u_{0}, x^{\alpha} ; n\right)+v_{1}\left(x^{\alpha}\right)
$$

avec $m$ fonctions scalaires $u^{I}$ et un vecteur $v_{1}$ que l'on peut choisir nul, où la phase $\varphi$ vérifie l'équation,

$$
\psi\left(u_{0}, x^{\alpha} ; \varphi_{\beta}\right)=0, \quad \psi\left(u, x^{\alpha} ; \varphi_{\beta}\right)=\varphi_{t}+|\operatorname{grad} \varphi| \lambda\left(u, x^{\alpha} ; n\right)
$$

Les rayons d'onde sont donnés par,

$$
\frac{d x^{\alpha}}{d \sigma}=\frac{\partial \psi_{0}}{\partial \varphi_{\alpha}}, \quad \frac{d \varphi_{\alpha}}{d \sigma}=-\frac{\partial \psi_{0}}{\partial x^{\alpha}}, \quad \psi_{0}\left(x^{\alpha} ; \varphi_{\beta}\right)=\psi\left(u_{0}\left(x^{\alpha}\right), x^{\alpha} ; \varphi_{\beta}\right) .
$$

C'est un système différentiel non linéaire pour les $2 n$ inconnues $x^{i}, \varphi_{i}$; il est inutile de considérer $\varphi_{0}$ qui ne figure pas dans les seconds membres et $x^{0}=\sigma$. Si $\left(x^{i}\right)_{\sigma=0}=x_{0}^{i}$, on se donnera la valeur de la phase à l'instant initial

$$
(\varphi)_{t=0}=\phi\left(x_{0}^{i}\right)
$$


et on aura ainsi, $\left(\varphi_{i}\right)_{i_{0}}=\phi_{i}\left(x_{0}^{j}\right)$ qui définira la normale $n_{0}$ au point $x_{0}^{i}$. Le systè me (8) fournira alors après intégration l'équation des rayons

$$
x^{i}=x^{i}\left(x_{0}^{j}, \sigma\right), \quad x^{i}\left(x_{0}^{j}, 0\right)=x_{0}^{i}, \quad t=\sigma .
$$

Si le jacobien

$$
J=D\left(x^{i}\right) / D\left(x_{0}^{i}\right)
$$

est fini et non nul on pourra inverser ces formules

$$
x_{0}^{i}=x_{0}^{i}\left(x^{j}, t\right), \quad \sigma=t
$$

et on aura,

$$
\varphi\left(x^{\alpha}\right)=\phi\left(x_{0}^{i}\left(x^{i}, t\right)\right)
$$

En prenant $v_{1}=0$ et en portant (6) dans (4) multipliée par $l_{I^{\prime}}\left(u_{0}, x^{\alpha} ; n\right)$ afin d'en chasser $u_{2}$, il vient,

$$
l_{I^{\circ} 0} A_{0}^{\alpha}\left(\frac{\partial u^{I}}{\partial x^{\alpha}} d_{I^{\circ}}+u^{I} \partial_{\alpha} d_{I^{\circ}}\right)+l_{I^{\circ}} \nabla A_{0}^{\alpha} u_{1}\left(u_{0 \alpha}+\varphi_{\alpha} \frac{\partial u^{I}}{\partial \xi} d_{I^{0}}\right)=l_{I^{\circ}} \nabla f_{0} u_{1} .
$$

D'une part [6],

$$
l_{I^{\prime} 0} A_{0}^{\alpha} d_{I^{0}}=\frac{\partial \psi_{0}}{\partial \varphi_{\alpha}} l_{I^{\prime}{ }_{0}} d_{I^{\circ}}
$$

et, d'autre part, en dérivant

$$
A^{\alpha} \varphi_{\alpha} d_{I}=\psi d_{I}
$$

dans la direction de $u_{1}$,

$$
\nabla A^{\alpha} \varphi_{\alpha} u_{1} d_{I}+\left(A^{\alpha} \varphi_{\alpha}-\psi I\right) \nabla d_{I} u_{1}=\left(\nabla \psi u_{1}\right) d_{I},
$$

d'où,

$$
l_{I^{\prime}} \nabla A^{\alpha} \varphi_{\alpha} u_{1} d_{I}=\left(\nabla \psi u_{1}\right) l_{I^{\prime}} d_{I}
$$

On peut récrire (11),

$$
\left\{\frac{\partial u^{I}}{\partial \sigma}+\left(\nabla \psi_{0} u_{1}\right) \frac{\partial u^{I}}{\partial \xi}\right\}\left(l_{I^{\prime}} d_{I}\right)_{0}+c_{I^{\prime} I}^{0} u^{I}=f_{I^{\prime} I}^{0} u^{I}, \quad u_{1}=u^{I} d_{I^{0}}
$$

où les coefficients

$$
c_{I^{\prime} I}^{0}=l_{I^{\circ},}\left(A_{0}^{\alpha} \partial_{\alpha} d_{I^{\circ}}+\nabla A_{0}^{\alpha} d_{I^{\circ}} u_{0 \alpha}\right), \quad f_{I^{\prime} I}^{0}=\left(l_{I^{\prime}}, \nabla f d_{I}\right)_{0}
$$


deviennent des fonctions de $\sigma$ par la substitution (9). On retrouve le système aux discontinuités en posant $\left.u^{I}=\xi \pi^{I}\left(x^{\alpha}\right){ }^{1}\right)$.

Les équations (12) ne sont pas linéaires sauf dans le cas exceptionnel,

$$
\nabla \lambda d_{I} \equiv 0
$$

En effet,

$$
\nabla \psi_{0} u_{1}=|\operatorname{grad} \varphi| \nabla \lambda_{0} d_{I^{\circ}} u^{I}
$$

Cependant (12) s'intègre par des quadratures lorsque l'onde est simple $(m=1)$. La non-linéarité provoque, comme l'a montré Mme Choquet, une distorsion du facteur de forme tandis que l'onde se propage sans déformation si elle est exceptionnelle [õ].

Beaucoup de systèmes de la physique sont, par chance, conservatifs, c'est-à-dire peuvent se mettre sous la forme,

$$
\partial_{\alpha} f^{\alpha}(u)=f\left(u, x^{\beta}\right), \quad f^{0}=u
$$

et leurs ondes multiples sont, en conséquence, exceptionnelles [7]. Il en résulte que (12) se résout encore facilement. De plus, on a,

$$
\begin{aligned}
& A^{\alpha}(u)=\nabla f^{\alpha}, \\
& \nabla A^{\alpha} d_{I} u_{\alpha}=\nabla \nabla f^{\alpha} d_{I} u_{\alpha}=\nabla \nabla f^{\alpha} u_{\alpha} d_{I}=\nabla A^{\alpha} u_{\alpha} d_{I}=\partial_{\alpha} A^{\alpha} d_{I},
\end{aligned}
$$

de sorte que,

$$
e_{I^{\prime} I}^{0}=l_{T^{\prime} 0} \partial_{\alpha}\left(A_{0}^{\alpha} d_{I^{0}}\right)
$$

qui se réduit simplement dans le cas unidimensionnel $(n=1)$ à

$$
c_{I^{\prime} I}^{0}=l_{Y^{\prime}}\left(\frac{\partial d_{I^{\mathrm{e}}}}{\partial \sigma}+\frac{\partial \lambda_{0}}{\partial x} d_{I_{0}}\right)
$$

\section{1. - Variation de l'élément de volume.}

Avant de poursuivre nous rappellerons une formule bien connue. On sait que le jacobien $J$ mesure le rapport des éléments de volume composés des mêmes particules à l'instant $t$ et à l'instant initial. En appliquant la règle de dérivation d'un

( $\left.{ }^{1}\right)$ On notera que $\partial \xi / \partial \sigma=\omega\left(\partial \psi_{0} / \partial \varphi_{\alpha}\right) \varphi_{\alpha}=\omega \psi_{0}=0$ d'après le théorème d'Euler sur les fonctions homogènes et (7). 
déterminant,

$$
\begin{aligned}
\frac{1}{J} \frac{\partial J}{\partial \sigma}=\frac{D\left(x_{0}^{i}\right)}{D\left(x^{i}\right)}\left\{\frac{D\left(\partial x^{1} / \partial \sigma, \ldots, x^{n}\right)}{D\left(x_{0}^{1}, \ldots, x_{0}^{n}\right)}+\ldots+\right. & \left.\frac{D\left(x^{1}, \ldots, \partial x^{n} / \partial \sigma\right)}{D\left(x_{0}^{1}, \ldots, x_{0}^{n}\right)}\right\}= \\
& =\frac{D\left(\Lambda_{0}^{1}, \ldots, x^{n}\right)}{D\left(x^{1}, \ldots, x^{n}\right)}+\ldots+\frac{D\left(x^{1}, \ldots, \Lambda_{0}^{n}\right)}{D\left(x^{1}, \ldots, x^{n}\right)}=\partial_{i} \Lambda_{0}^{i},
\end{aligned}
$$

où les $\Lambda_{0}^{i}=\partial \psi_{0} / \partial \varphi_{i}$ sont les composantes de la vitesse radiale. Pour la suite il est utile d'introduire

$$
\theta\left(\sigma, x_{0}^{i}\right)=\sqrt{\mathcal{J}}
$$

\section{2. - Solution de base constante.}

Un premier cas intéressant est celui où $u_{0}=$ Cte ce qui suppose que $f\left(u_{0}, x^{\alpha}\right)=0$. Si les matrices $A^{\alpha}$ ne dépendent pas explicitement des coordonnées on voit immédiatement d'après (8) que les $\varphi_{i}$ sont constants le long des rayons et que

$$
x^{i}=x_{0}^{i}+\Lambda^{i}\left(u_{0}, n_{0}\right) \sigma,
$$

tandis que (13) fournit [6],

$$
e_{I^{\prime} Y}^{0}=l_{I^{\prime} 0} A_{0}^{\alpha} \frac{\partial d_{T_{0}}}{\partial \varphi_{\beta}} \varphi_{\alpha \beta}=\left(l_{I^{\prime}} d_{I}\right)_{0} \frac{\partial}{\partial \sigma} \log \theta
$$

$\theta^{2}$ est un polynôme de degré $n-1$ au plus en $\sigma$ et s'exprime à l'aide des courbures totale et moyenne de la surface $\phi\left(x_{0}^{i}\right)=$ Cte lorsque la vitesse $\lambda_{0}$ est indépendante de $n[6],[8]$.

Si l'onde est simple, (12) fournit l'équation,

$$
\begin{aligned}
& \frac{\partial u^{1}}{\partial \sigma}+(\nabla \psi d)_{0} u^{1} \frac{\partial u^{1}}{\partial \xi}+\frac{1}{\theta} \frac{\partial \theta}{\partial \sigma} u^{1}=v_{0} u^{1}, \\
& u_{1}=u^{1} d_{0}, \quad v_{0}=(l \nabla f d / l d)_{0},
\end{aligned}
$$

qui se ramène à $[9]$,

$$
\frac{\partial v}{\partial \tau}+v \frac{\partial v}{\partial \xi}=0
$$

avec

$$
u^{1}=\frac{v}{\theta} e^{w}, \quad \tau=\int_{0}^{\sigma}(\nabla \psi d)_{0} \frac{e^{w}}{\theta} d \sigma, \quad w=\int_{0}^{\sigma} v_{0} d \sigma
$$


En particulier si le second membre de (1) ne dépend que de $u, w=\nu_{0} \sigma$. On se donne à l'instant initial,

$$
u^{1}=F\left(x_{0}^{i} ; \xi_{0}\right), \quad \xi_{0}=\omega \phi
$$

et $v$ est déterminé par l'équation implicite,

$$
v=F\left(x_{0}^{i} ; \xi-v \tau\right)
$$

qui met en évidence la đistorsion de l'onde ${ }^{(1)}$. Les singularités de $u$ apparaîtront quand $\theta=0$ (caustique) ou lorsque la dérivée de (20) par rapport à $v$ sera nulle,

$$
1+\tau \frac{\partial F}{\partial \xi_{0}}\left(x_{0}^{i} ; \xi-v \tau\right)=0 .
$$

$\mathrm{Au}$ contraire si l'onde est exceptionnelle, on aura simplement,

$$
u^{I}=\frac{1}{\theta} w_{\Gamma^{\prime}}^{I}\left(x_{0}^{i}, \sigma\right) F^{I^{\prime}}\left(x_{0}^{i} ; \xi\right)
$$

où les coefficients $w_{I^{\prime}}^{I}$ vérifient,

$$
\left(l_{I^{\prime}} d_{I}\right)_{0}\left(\partial w_{I^{\prime}}^{I} / \partial \sigma\right)=f_{I^{\prime} I}^{0} w_{I^{*}}^{I}, \quad\left(w_{I^{\prime}}^{I}\right)_{\sigma=0}=\delta_{I^{\prime}}^{I},
$$

système différentiel à coefficients constants si $f=f(u)$. La substitution de $\left(9^{\prime}\right)$ permet alors d'écrire les $u^{I}$ en fonction des $x^{\alpha}$.

\section{3. - Systèmes à intégrale d'énergie.}

Un système conservatif (14),

$$
\partial_{t} u+\partial_{j} f^{j}(u)=f\left(u, x^{\beta}\right)
$$

qui admet une intégrale d'énergie

$$
\partial_{\alpha} h^{\alpha}(u)=g\left(u, x^{\beta}\right), \quad h^{0}=h,
$$

où $h(u)$ est une fonction convexe (le hessien $H=\nabla \breve{V} h$ est une matrice $N \times N$ définie positive; "indique la transposition) peut se mettre sous une forme symétrique [10]

(') En prenant $F\left(x_{0}^{i} ; \xi_{0}\right)=\xi_{0} \pi\left(x_{0}^{i}\right)$ on retrouve la loi d'évolution des discontinuités du premier ordre (des dérivées premières) [6]. 
et même peut s'écrire [11] (1),

$$
A^{\prime \alpha} u_{\alpha}^{\prime}=f, \quad A^{\prime \alpha}=\nabla^{\prime} \breve{\nabla}^{\prime} h^{\prime \alpha}, \quad h^{\prime 0}=h^{\prime}, \quad A^{\prime 0}=H^{\prime}=H^{-1}
$$

où les

$$
h^{\prime \alpha}=\breve{u}^{\prime} f^{\alpha}-h^{\alpha}
$$

sont $n+1$ fonctions des variables nouvelles de champ,

$$
u^{\prime}=\breve{\nabla} h \text {. }
$$

Entre les matrices $A^{\alpha}$ et $A^{\prime \alpha}$ et les vecteurs propres existent les relations,

$$
A^{\prime \alpha}=A^{\alpha} H^{\prime}, \quad d_{I}^{\prime}=H d_{I}, \quad l_{I}^{\prime}=l_{I} .
$$

De plus, en raison de la symétrie des matrices $A^{\prime \alpha}$,

$$
\breve{l}_{I}^{\prime}=d_{I}^{\prime}
$$

et si l'on impose, ce qui est toujours possible puisque le système de vecteurs propres est complet, la condition,

$$
l_{I} d_{I^{\prime}}=l_{I}^{\prime} H^{\prime} d_{I^{\prime}}^{\prime}=\delta_{I I^{\prime}},
$$

ces vecteurs seront orthonormés par $H$,

$$
\breve{d}_{I^{\prime}} H d_{I}=\delta_{I^{\prime} I} .
$$

Il reste encore un certain arbitraire pour les vecteurs propres. Donnons-nous $m$ vecteurs propres indépendants $\bar{d}_{\bar{I}}$ et posons

$$
\Delta_{I^{\prime} I}=\breve{\bar{d}}_{I^{\prime}} H \bar{d}_{I} .
$$

Le changement

$$
\bar{d}_{I^{\prime}}=\alpha_{I^{\prime}}^{I} d_{I}, \quad \operatorname{det} \alpha=\operatorname{det}\left(\alpha_{I^{\prime}}^{I}\right) \neq 0
$$

et (28) impliquent

$$
\Delta=\alpha \check{\alpha}
$$

( $\left.{ }^{1}\right)$ On notera que les matrices $A^{i}$ ne peuvent être symétriques si les composantes de $u$ n'ont pas toutes la même dimension physique. En effet, si elles le sont, il existe la densité d'énergie $h=\frac{1}{2} \breve{u} u$ et les termes de cette somme doivent évidemment avoir la même dimension. 
Comme $\Delta=\left(\Delta_{I^{\prime} I}\right)$ est une matrice $(m \times m)$ symétrique définie positive, il est toujours possible de trouver $\alpha$ telle que cette condition soit vérifiée. Mais on voit que $\alpha T$ convient aussi, où $T$ est une matrice unitaire: $\breve{T}=T^{-1}$. Bien entendu, si $m=1$, le vecteur propre $d$ sera défini au signe près par (28).

\section{4. - Equations variationnelles.}

A partir d'un lagrangien,

$$
L=L\left(q_{\alpha}^{s}, q^{s}\right), \quad q_{\alpha}^{s}=\partial_{\alpha} q^{s}\left(x^{\beta}\right), \quad s=1,2, \ldots, \bar{N},
$$

on déduit les équations d'Euler,

$$
\partial_{\alpha}\left(\frac{\partial L}{\partial q_{\alpha}^{s}}\right)-\frac{\partial L}{\partial q^{s}}=\mathbf{0}
$$

que l'on peut mettre sous la forme (14) avec (1),

$$
u=\left[\begin{array}{c}
\frac{\partial L}{\partial q_{0}^{s}} \\
q_{i}^{s} \\
q^{s}
\end{array}\right]=\left[\begin{array}{c}
u_{s}^{0} \\
u_{i}^{s} \\
u^{s}
\end{array}\right], \quad f^{j}=\left[\begin{array}{c}
\frac{\partial L}{\partial q_{j}^{s}} \\
-q_{0}^{s} \delta_{i}^{j} \\
0
\end{array}\right], \quad f=\left[\begin{array}{c}
\frac{\partial L}{\partial q^{s}} \\
0 \\
q_{0}^{s}
\end{array}\right]
$$

où la seconde colonne indique les noms des composantes de $u$. Les indices $s$ et $i$ sont muets et $u$ a $N=(n+2) \bar{N}$ composantes tandis que les zéros qui figurent dans $f^{j}$ et $f$ représentent respectivement $\bar{N}$ et $n \bar{N}$ zéros.

Il s'y ajoute les contraintes,

$$
\partial_{i} u^{s}-u_{i}^{s}=0
$$

qui ne font intervenir que des dérivées spatiales de $u$ et qui sont vraies à un instant quelconque pourvu qu'elles le soient à l'instant initial. En effet,

$$
\partial_{t}\left(\partial_{i} u^{s}-u_{i}^{s}\right)=\partial_{i}\left(\partial_{t} u^{s}\right)-\partial_{i} u_{i}^{s}=\partial_{i} q_{0}^{s}-\hat{o}_{i} q_{0}^{s}=0
$$

compte tenu des équations d'évolution (30).

Si l'on écrit le développement (2),

$$
u_{i}^{s}=u_{i(0)}^{s}+\frac{1}{\omega} u_{i(1)}^{s}+\ldots, \quad u^{s}=u_{(0)}^{s}+\frac{1}{\omega} u_{(1)}^{s}+\frac{1}{\omega^{2}} u_{(2)}^{s}+\ldots,
$$

(1) Si $L=L\left(q_{\alpha}^{s}\right)$, on ne fera pas figurer $q^{s}$ dans $u$ et $f$ sera nul. 
on voit tout de suite que les contraintes imposent,

$$
\frac{\partial u_{(1)}^{s}}{\partial \xi}=0
$$

c'est-à-dire (avec le choix $v_{1}=0$ dans l'équation (6)) $u_{(1)}^{s}=0$ ainsi que

$$
u_{\xi(1)}^{s}=\varphi_{i} \frac{\partial u_{(2)}^{s}}{\partial \xi},
$$

conditions à satisfaire par les données initiales.

Il est facile de vérifier l'existence de (23) où,

$$
h=q_{0}^{s} \frac{\partial L}{\partial q_{0}^{s}}-L, \quad h^{i}=q_{0}^{s} \frac{\partial L}{\partial q_{i}^{s}}, \quad g=0
$$

ef on supposera, en plus de l'byperbolicité de (29), que $h$ est convexe comme toute bonne densité d'énergie.

Il vient alors,

$$
u^{\prime}=\left[\begin{array}{c}
q_{0}^{s} \\
-\frac{\partial L}{\partial q_{i}^{s}} \\
-\frac{\partial L}{\partial q^{s}}
\end{array}\right]=\left[\begin{array}{c}
u_{0}^{\prime s} \\
u_{s}^{\prime i} \\
u_{s}^{\prime}
\end{array}\right], \quad f^{j}=\left[\begin{array}{c}
-u_{s}^{\prime s} \\
-u_{0}^{\prime s} \delta_{i}^{j} \\
0
\end{array}\right], \quad f=\left[\begin{array}{c}
-u_{s}^{\prime} \\
0 \\
u_{0}^{\prime s}
\end{array}\right]
$$

puis, d'après (25),

$$
h^{\prime}=L-q_{i}^{s} \frac{\partial L}{\partial q_{i}^{s}}-q^{s} \frac{\partial L}{\partial q^{s}}, \quad h^{\prime i}=h^{i}=-u_{0}^{\prime s} u_{s}^{\prime i} .
$$

On déduit immédiatement de (31) ou (32) que

$$
A^{\prime i}=\text { Cte }
$$

et comme $f$ est fonction linéaire de $u^{\prime}$ la seule non-linéarité de (24) résidera dans le coefficient $H^{\prime}\left(u^{\prime}\right)$ de la dérivée temporelle,

$$
H^{\prime}\left(u^{\prime}\right) u_{t}^{\prime}+A^{\prime i} u_{i}^{\prime}=B^{\prime} u^{\prime}, \quad \breve{H}^{\prime}=H^{\prime}, \quad \breve{A}^{\prime i}=A^{\prime i}
$$

et $B^{\prime}$ est une matrice antisymétrique. En effet,

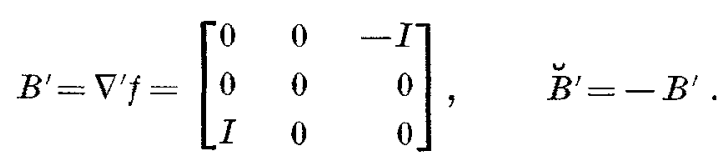


Passons maintenant au calcul des coefficients de (12). D'après (15), (26),

$$
\begin{gathered}
c_{I^{\prime} I}=l_{I^{\prime}} \partial_{\alpha}\left(A^{\alpha} d_{I}\right)=l_{I^{\prime}}^{\prime} \partial_{\alpha}\left(A^{\prime \alpha} d_{I}^{\prime}\right)=l_{I^{\prime}}^{\prime} \partial_{t}\left(H^{\prime} d_{I}^{\prime}\right)+l_{I^{\prime}}^{\prime} A^{\prime i} \partial_{i} d_{I}^{\prime}=a_{I^{\prime} I}+b_{I^{\prime} I}+l_{I^{\prime}}^{\prime} \partial_{t} H^{\prime} d_{I}^{\prime}, \\
a_{I^{\prime} I}=l_{I^{\prime}}^{\prime} A^{\prime i} \partial_{i} d_{I}^{\prime}, \quad b_{I^{\prime} I}=l_{I^{\prime}}^{\prime} H^{\prime} \partial_{t} d_{I}^{\prime} .
\end{gathered}
$$

En vertu de (27),

$$
\partial_{t} l_{I^{\prime}}^{\prime} H^{\prime} d_{I}^{\prime}+l_{I^{\prime}}^{\prime} H^{\prime} \partial_{t} d_{I}^{\prime}+l_{I^{\prime}}^{\prime} \partial_{t} H^{\prime} d_{I}^{\prime}=0
$$

et, par transposition,

$$
\partial_{t} l_{I^{\prime}}^{\prime} H^{\prime} d_{I}^{\prime}=l_{I}^{\prime} \boldsymbol{H}^{\prime} \partial_{t} d_{I^{\prime}}^{\prime}
$$

si bien que

$$
b_{\left(I I^{\prime}\right)}=-\frac{1}{2} l_{I^{\prime}}^{\prime} \partial_{b} H^{\prime} d_{I}^{\prime}
$$

et

$$
c_{I^{\prime} T}=a_{I^{\prime} I}-b_{I I^{\prime}}
$$

En dérivant l'identité,

$$
\left(A_{n}^{\prime}-\lambda H^{\prime}\right) d_{I}^{\prime}=0
$$

par rapport à $u^{\prime}$,

$$
\left(A_{n}^{\prime}-\lambda H^{\prime}\right) \nabla^{\prime} d_{I}^{\prime}-H^{\prime} d_{I}^{\prime} \nabla^{\prime} \lambda-\lambda \nabla^{\prime} H^{\prime} d_{I}^{\prime}=0
$$

d'où,

$$
\lambda l_{I^{\prime}}^{\prime} \nabla^{\prime} H^{\prime} d_{I}^{\prime}+\delta_{I^{\prime} I} \nabla^{\prime} \lambda=0
$$

et, en multipliant par $u_{t}^{\prime}$,

$$
b_{\left(I I^{\prime}\right)}^{0}=\frac{1}{2 \lambda} \nabla^{\prime} \lambda u_{t}^{\prime} \delta_{I I^{\prime}}=\frac{1}{2 \lambda} \frac{\partial \lambda}{\partial t} \delta_{I I^{\prime}}
$$

la dérivée temporelle de $\lambda(u ; n)$ étant prise à $n$ constant.

D'autre part,

$$
\partial_{\alpha}\left(l_{I^{\prime}}^{\prime} A^{\prime \alpha} d_{I}^{\prime}\right)=\partial_{\alpha}\left(\frac{\partial \psi}{\partial \varphi_{\alpha}}\right) \delta_{I^{\prime} I}=\partial_{i}\left(l_{I^{\prime}}^{\prime} A^{\prime i} d_{I}^{\prime}\right)=a_{I^{\prime} I}+a_{I I^{\prime}},
$$

c'est-à-dire,

$$
a_{\left(I I^{\prime}\right)}=\frac{1}{2} \operatorname{div} A \delta_{I I^{\prime}}
$$

soit, en raison de (16), (35),

$$
\hat{e}_{\left(I I^{\prime}\right)}^{0}=\frac{1}{2}\left(\operatorname{div} \Lambda_{0}-\frac{1}{\lambda_{0}} \frac{\partial \lambda_{0}}{\partial t}\right) \delta_{I I^{\prime}}=\left(\frac{\partial}{\partial \sigma} \log \theta-\frac{1}{2 \lambda_{0}} \frac{\partial \lambda_{0}}{\partial t}\right) \delta_{I I^{\prime}}
$$


Enfin,

$$
f_{I^{\prime} I}=l_{I^{\prime}} \nabla f d_{I}=l_{I^{\prime}}^{\prime} B^{\prime} d_{I}^{\prime}=\breve{d}_{I}^{\prime} \breve{B}^{\prime} \breve{l}_{I^{\prime}}^{\prime}=-l_{I}^{\prime} B^{\prime} d_{I^{\prime}}^{\prime}=-f_{I I^{\prime}},
$$

soit

$$
f_{\left(I I^{\prime}\right)}^{0}=0
$$

Le système (12) devient done,

$$
\left\{\frac{\partial u^{I}}{\partial \sigma}+|\operatorname{grad} \varphi|\left(\nabla \lambda_{0} u_{1}\right) \frac{\partial u^{I}}{\partial \xi}\right\} \delta_{I^{\prime} I}+c_{I^{\prime} I}^{0} u^{I}=f_{I^{\prime} I}^{\mathrm{n}} u^{I}
$$

On pose,

$$
\left|u_{1}\right|=\sqrt{\sum_{1}^{m}\left(u^{I}\right)^{2}}
$$

et on se donne

$$
\left|u_{1}\right|_{t=0}=F\left(x_{0}^{i} ; \xi_{0}\right) .
$$

Si l'onde n'est pas exceptionnelle, done simple, $\left|u_{1}\right|$ est fourni par (16), (19), (20),

$$
\left|u_{1}\right|=\frac{v}{\theta} e^{w}, \quad v=F\left(x_{0}^{i} ; \xi-v \tau\right)
$$

avec maintenant,

$$
w=\int_{0}^{\sigma} \frac{1}{2 \lambda_{0}} \frac{\partial \lambda_{0}}{\partial t} d \sigma
$$

Si l'onde, au contraire, est exceptionnelle, on obtiendra, en multipliant (39) par $u^{I^{\prime}}$, une équation linéaire dont la solution est,

$$
\left|u_{1}\right|=F\left(x_{0}^{i} ; \xi\right) \frac{e^{w}}{\theta}
$$

où $w$ est toujours donné par (42). $w$ sera une fonction croissante de $\sigma$ si $\partial\left|\lambda_{0}\right| / \partial t>0$ Si $u_{0}$ est un champ stationnaire, $w=0$, tandis que $u_{0}=u_{0}(t)$ entraîne, d'après $(8)$, $n\left(x^{i}\right)=n\left(x_{0}^{i}\right)$ et $\partial \lambda_{0} / \partial \sigma=\partial \lambda_{0} / \partial t$, d'où il découle,

$$
e^{u n}=\sqrt{\frac{\lambda_{0}\left(u_{0}(\sigma) ; n\right)}{\lambda_{0}\left(u_{0}(0) ; n\right)}}
$$

Les expressions (41), (43) restent valables pour un choix quelconque des vecteurs propres droits à condition de définir (de manière intrinsèque) la norme de $u_{1}$ par,

$$
\left|u_{1}\right|=\left(\breve{u}_{1} H_{0} u_{1}\right)^{\frac{1}{2}}
$$

qui, avec le choix (28), se réduit bien à (40). 
Pour terminer nous rappellerons que le critère d'exceptionnalité s'exprime d'une manière covariante [12] commode à utiliser quand les équations du champ ont ellesmêmes une forme covariante.

Spécialement intéressants sont, d'autre part, les systèmes conservatifs strictement exceptionnels [13] pour lesquels les seules surfaces de choc possibles sont les surfaces d'ondes (caractéristiques). Cette classe comprend évidemment tous les systèmes linéaires mais est plus restreinte que celle des systèmes complètement exceptionnels (1).

Note ajoutée sur les épreuves. - Les systèmes de la forme (24) ont été introduits par Godunov [15] sur trois exemples dont, en particulier, les équations d'Euler pour $L=L\left(q_{\alpha}^{s}\right)\left(^{(2}\right)$.

En posant $\varrho=|\operatorname{grad} \varphi| \lambda$, l'équation (8) correspondant à $\varphi_{t}$ montre immédiatement que

$$
\frac{1}{\lambda_{0}} \frac{\partial \lambda_{0}}{\partial t}=\frac{d}{d \sigma} \log |\varrho|,
$$

de sorte que (42) donne simplement,

$$
e^{w}=\sqrt{\left|\frac{\varrho(\sigma)}{\varrho(0)}\right|} .
$$

( ${ }^{1}$. Les ondes du fluide relativiste qui a l'équation d'état:

$$
\text { densité d'énergie }=\text { pression }+ \text { fonction de l'entropie, }
$$

sont toutes exceptionnelles mais la fonction doit se réduire à une constante pour que le système, soit strictement exceptionnel [14].

(2) Nous remercions M. FABRIzio de nous avoir signalé cet article.

\section{BIBLIOGRAPHIE}

[1] R. Codrant - D. Hilbert, Methods of Mathematical Physics, vol. II, Interscience Publ. New York (1962).

[2] J. Leray, Cahiers Phys., 15 (1961), p. 373; L. GArding - T. Kotake - J. Leray, Bull. Soc. Math. Fr., 92 (1964), p. 263.

[3] P. D. LAX, Duke Math. Journ., 24 (1957), p. 627.

[4] D. Ludwig, Comm. Pure Appl. Math., 13 (1960), p. 473. Voir aussi [1].

[5] Y. Choquet-Bruhat, J. Math. pures appl., 48 (1969), p. 117; Y. Choquet-Bruhat, Couplage d'ondes gravitationnelles et électromagnétiques à haute fréquence, in Ondes et radiations gravitationnelles, colloque C.N.R.S., Paris (juin 1973). Editions du C.N.R.S., Paris. L. Lamoureux, Ondes asymptotiques dans les milieux élastiques relativistes, Ibid.

[6] G. BoIllat, La propagation des ondes, Gauthier-Villars, Paris (1965).

[7] G. Borllat, C. R. Acad. Se. Paris, 274 A (1972), p. 1018.

[8] T. Y. Thomas, Journ. Math. Mech., 6 (1957), p. 455.

[9] T. TANidTi, Progr. Theor. Phys., Suppl. no. 55 (1974), p. 1, obtient une équation similaire dans le cas unidimensionnel en faisant un changement des variable $x, t$ aussi utilisé 
par C. S. Gardner - G. K. Morikawa, Comm. Pure Appl. Math., 18 (1965), p. 35. Voir aussi, A. JFFFrey - T. KaKUTANI, SIAM Review, 14 (1972), p. 582 et les articles de R. M. MuUra et G. B. WhImHAM dans Nonlinear Waves, edited by \$. Leibovich * A. R. Seebass, Cornell University Press, Ithaca-London (1974), ainsi que le supplément no. 5ธ cité plus haut.

[10] K. O. Friedrichs - P. D. Lax, Proe. Nat. Acad. Sci. USA, 68 (1971), p. 1686.

[11] G. Borllat, C. R. Acad. Se. Paris, 278 A (1974), p. 909; 280 A (1975), p. 1325.

[12] G. Bonllat, Journ. Math. Phys., 14 (1973), p. 973.

[13] G. Borldat, Phys. Lett., 50 A (1974), p. 357.

[14] G. Bonlati, C. R. Acad. Se. Paris, 275 A (1972), p. 1255.

[15] S. K. Godunov, Sov. Math., 2 (1961), p. 947. 